\title{
UNCERTAINTY ANALYSIS OF DATA-BASED MODELS FOR ESTIMATING COLLAPSE MOMENTS OF WALL-THINNED PIPE BENDS AND ELBOWS
}

\author{
DONG SU KIM ${ }^{1}$, JU HYUN KIM ${ }^{2}$, MAN GYUN NA ${ }^{2, *}$, and JIN WEON KIM ${ }^{2}$ \\ ${ }^{1}$ Korea Atomic Energy Research Institute \\ Daedeok-daero, Yuseong-gu, Daejeon 305-348, Republic of Korea \\ ${ }^{2}$ Department of Nuclear Engineering, Chosun University \\ 375 Seosuk-dong, Dong-gu, Gwangju 501-759, Republic of Korea \\ *Corresponding author. E-mail : magyna@chosun.ac.kr
}

Received June 13, 2011

Accepted for Publication July 14, 2011

The development of data-based models requires uncertainty analysis to explain the accuracy of their predictions. In this paper, an uncertainty analysis of the support vector regression (SVR) model, which is a data-based model, was performed because previous research showed that the SVR method accurately estimates the collapse moments of wall-thinned pipe bends and elbows. The uncertainty analysis method used in this study was an analytic uncertainty analysis method, and estimates with a 95\% confidence interval were obtained for 370 test data points. From the results, the prediction interval (PI) was very narrow, which means that the predicted values are quite accurate. Therefore, the proposed SVR method can be used effectively to assess and validate the integrity of the wall-thinned pipe bends and elbows.

KEYWORDS : Collapse Moment, Uncertainty Analysis, Support Vector Regression, Wall-thinned Defect

\section{INTRODUCTION}

The pipe bends and elbows are considered to be critical components in the piping systems of nuclear power plants because they are incorporated into the piping systems to allow modification of isometric routing and reduce anchor reaction forces. In addition, the pipe bends and elbows can absorb substantial thermal expansion and seismic movement through the energy dissipation incurred as a result of local plastic deformation. Therefore, they can maintain the integrity of the piping system under transiently loading conditions [1-2]. On the other hand, care must be taken to ensure that the collapse load is avoided. Therefore, it is essential to accurately estimate the safety margin for the collapse of pipe bends and elbows under various ranges of operating conditions.

On the other hand, the pipe bends and elbows in nuclear power plants face a variety of degradation mechanisms. In particular, wall-thinning degradation is considered to be an important degradation mechanism in carbon steel elbows [3]. The wall-thinned defect is caused mainly by flow-accelerated corrosion, which reduces the failure pressure, load-carrying capacity, deformation ability, and fatigue resistance of pipe bends and elbows. Therefore, as mentioned above, it is essential to examine the effect of wall-thinned defects on the failure behavior of pipe bends and elbows and to accurately estimate the collapse loads of wall-thinned bends and elbows under a range of loading conditions.

To train and test the data-based models, the collapse moment-related data should be provided. The data was obtained by finite element analyses (FEAs) under various loading conditions and defect geometries as follows: the thinning defect locations of extrados, intrados, and the crown; bend radius; bend angle; wall thickness at the thinning defect; thinning length; thinning angle; internal pressure; and bending modes of closing and opening.

In previous studies, data-based models, such as the support vector regression (SVR) and fuzzy neural network method (FNN), were applied successfully to the estimation of collapse moments [4-5]. On the other hand, uncertainty analysis was not performed for the developed models. This paper will build on the SVR method, which shows the better performance of the two models, to analyze the uncertainty of the collapse moment estimates and will use analytical uncertainty analysis methods to predict the prediction interval (PI). In addition, this paper deals partly with the SVR method used to estimate the collapse moments. 


\section{COLLAPSE MOMENT CALCULATION USING FINITE ELEMENT ANALYSES}

This study employed the collapse moment calculated using the twice-elastic slope method, as illustrated in Fig. 1 [6-7]. Carbon steel bends with an outer diameter $\left(D_{o}\right)$ of $400 \mathrm{~mm}$ and a nominal thickness $\left(t_{\text {nom }}\right)$ of $20 \mathrm{~mm}$ were used. The bend angles $(\phi)$ were $30^{\circ}, 60^{\circ}$, and $90^{\circ}$, and the bend radius ratios $\left(R_{b} / R_{m}\right)$ were 3 and 6 , respectively (refer to Fig. 2). The bends and elbows were connected to straight pipes with lengths equal to ten times the mean radius $\left(R_{m}\right)$ to allow free ovalization of the end section of the bends. The wall-thinning defects were located at the intrados and extrados centerlines as well as at the crown of the pipe bends and elbows. The axial and circumferential shapes of the defects were circular.

Nonlinear three-dimensional finite element analysis (FEA) was carried out to evaluate the collapse moment of wall-thinned pipe bends. Fig. 3 depicts the finite element meshes used in the analysis. Twenty node solid elements with a reduced integration order were used to model the bend and pipes. Considering geometrical symmetry, onefourth of the bend is modeled for the intrados and extrados

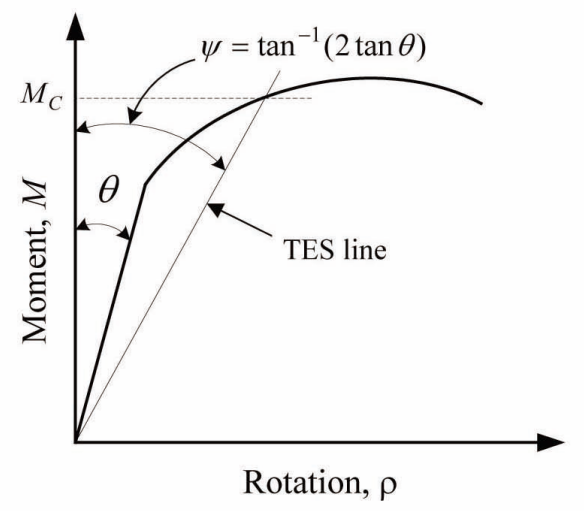

(a) Case 1

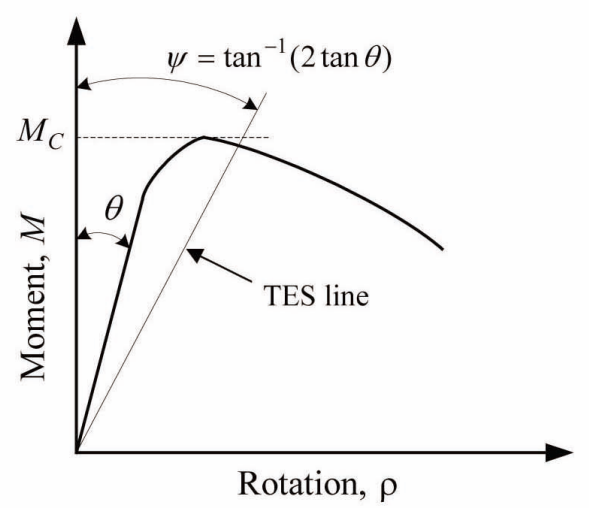

(b) Case 2

Fig. 1. Illustration of the Collapse Moment Definition [5]. wall-thinning defects, and half of the bend is modeled for a crown wall-thinning defect [4-5]. In FEA, various loading conditions and defect geometries were considered, as summarized in Table 1. The combined internal pressure and bending loads were considered as an applied load. A range of internal pressures from 0 to $20 \mathrm{MPa}$ was used, and the in-plane bending of either the closing- or opening-mode was applied at a constant internal pressure.

The deformation behavior of an elbow under a bending load is governed by its geometry, including the bend radius, bend angle, and radius/thickness $(R / t)$. Therefore, the collapse behavior of a local wall-thinned elbow is also affected by these geometrical parameters. The $R / t$ of an elbow is dependent on the safety class and operating pressure of the piping system, and elbows with $R / t$ values of 10 to 30 are typically used in the secondary piping system of a NPP, at which local wall-thinning is more

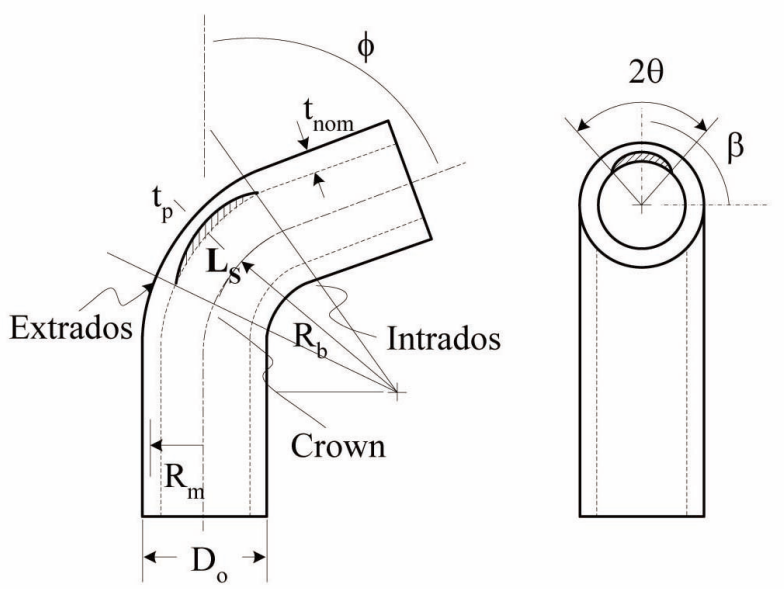

Fig. 2. Definition of the Dimensions for Wall-thinned Defects in Pipe Bends [5].

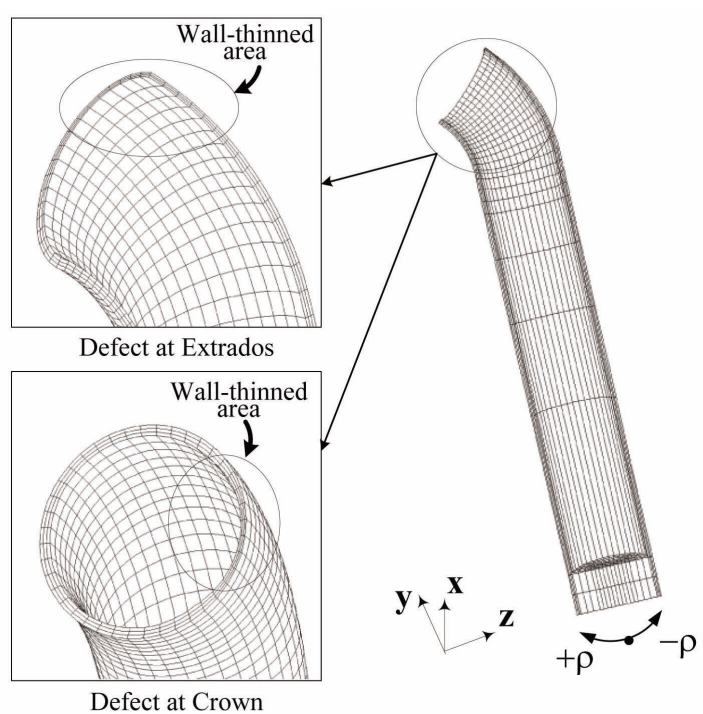

Fig. 3. Finite Element Model used in FEAs [5]. 
likely to occur. As the present results were developed based on the analysis results of an elbow when $R / t=10$, the results are applicable to an evaluation of wall-thinned elbows in a secondary piping system operated at relatively high pressures, i.e. a high-energy piping system. Moreover, additional analysis and verification considering various $\mathrm{R} / \mathrm{t}$ values will be needed to expand the applicability of the present results to integrity evaluations of wall-thinned elbows operated at moderate and low pressures.

The general-purpose FEA program, ABAQUS [8] was used in this study. A former FEA evaluation showed that consideration of the geometrical nonlinearity in the FEA is quite important for a precise determination of a pipe bend deflection under a range of combinations of closing and opening modes of bending and internal pressure [9]. Therefore, both geometric and material nonlinearity were considered in this analysis. The yield stress and ultimate tensile stress of the specific material of the bend and the attached pipes were $302 \mathrm{MPa}$ and $450 \mathrm{MPa}$, and the elastic modulus and Poisson ratio were 206GPa and 0.3, respectively. Fig. 4 shows the true stress-true strain curve used in the analysis.

Table 1. FEA Conditions for Wall-thinned Pipe Bends [5].

\begin{tabular}{c|c|c}
\hline \multicolumn{2}{c|}{ Wall-thinned location of pipe bends } & Extrados, Intrados, Crown \\
\hline \multicolumn{2}{c}{ Bend radius $\left(\mathrm{R}_{\mathrm{b}} / \mathrm{R}_{\mathrm{m}}\right)$} & 3,6 \\
\hline \multicolumn{2}{c}{ Bending angle $\left({ }^{\circ}\right)$} & $30,60,90$ \\
\hline \multirow{3}{*}{ Defect geometry } & $L_{s} / D_{o}$ & $0.25,0.5,1.0,1.5,2.0$ \\
\cline { 2 - 3 } & $\left(t_{\text {nom }}-t_{p}\right) / t_{\text {nom }}$ & $0.233,0.466,0.699$ \\
\cline { 2 - 3 } Load & $\theta / \pi$ & $0.0625,0.125,0.25,0.50$ \\
\hline \multirow{2}{*}{ Bending mode } & Closing, Opening \\
\cline { 2 - 3 } & Pressure $(\mathrm{MPa})$ & $0,5,10,15,20$ \\
\hline
\end{tabular}

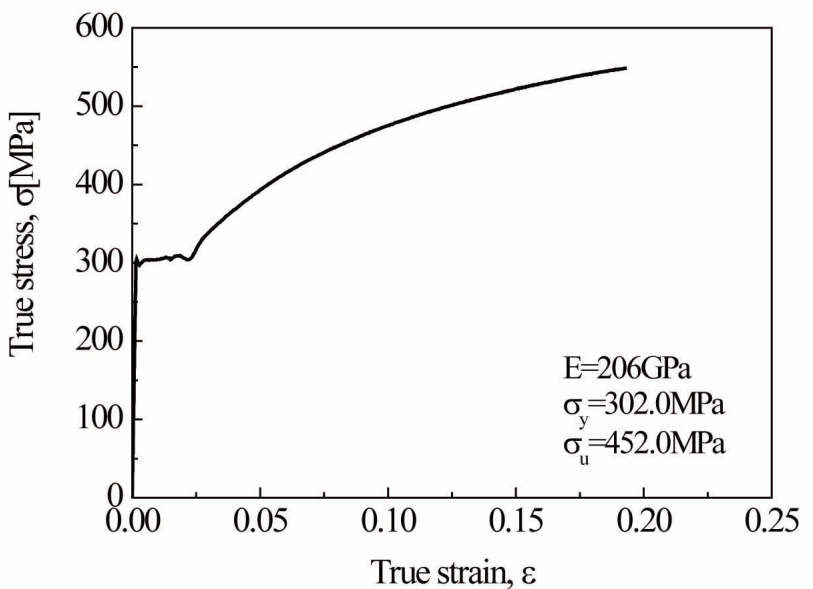

Fig. 4. True Stress Versus True Strain Curve used in FEAs [5].

\section{SUPPORT VECTOR REGRESSION}

\subsection{Model Development}

In general regression learning problems, the learning machine is given training data from which it attempts to learn the input-output relationship. The SVR first maps the original input data $\mathbf{x}$ into high dimensional feature space by nonlinear mapping. The unknown function is then solved by determining the coefficients of the basis function of the linear expansion. Therefore, for a given set of data $\left\{\left(\mathbf{x}_{i}, y_{i}\right)\right\}_{i=1}^{N}$, where $\mathbf{x}_{i}$ is the input vector to an SVR model, $y_{i}$ is the actual output value, and $N$ is the total number of data, the support vector approximation can be expanded as follows:

$$
y=f(\mathbf{x})=\sum_{i=1}^{N} w_{i} \phi_{i}(\mathbf{x})=\mathbf{w}^{T} \boldsymbol{\varphi}(\mathbf{x})+b,
$$

where $\phi_{i}(\mathbf{x})$ is called the feature that is nonlinearly mapped from the input space $\mathbf{x}, \mathbf{w}=\left[w_{1} w_{2} \cdots w_{N}\right]^{T}$, and $\boldsymbol{\varphi}=\left[\phi_{1} \phi_{2}\right.$

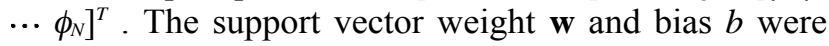
calculated by minimizing the following regularized risk function:

$$
R(\mathbf{w})=\frac{1}{2} \mathbf{w}^{T} \mathbf{w}+\lambda \sum_{i=1}^{N} L_{\varepsilon}\left(\mathbf{x}_{i}, y_{i}, f\right),
$$

where

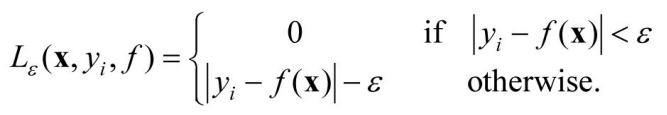

Here, parameters $\lambda$ and $\varepsilon$ are defined by the user. The first term of Eq. (2) characterizes the complexity of the SVR model, and $L_{\varepsilon}\left(\mathbf{x}, y_{i}, f\right)$ is called the $\varepsilon$-insensitive loss function [10]. Note that the parameter $\lambda$ affects the tradeoff between the approximation error and the weights vector norm. The loss is equal to zero if the difference between the estimated $f(\mathbf{x})$ and measured values is less than the error level $\varepsilon$. For all other estimated points outside the error level, $\varepsilon$, the loss is equal to the magnitude of the difference between the estimated value and $\varepsilon$ (refer to Fig. 5).

The regularized risk function can be rewritten using the following constrained form:

$$
R\left(\mathbf{w}, \xi, \xi^{*}\right)=\frac{1}{2} \mathbf{w}^{T} \mathbf{w}+\lambda \sum_{i=1}^{N}\left(\xi_{i}+\xi_{i}^{*}\right)
$$

subject to the constraints $\left\{\begin{array}{l}y_{i}-\mathbf{w}^{T} \boldsymbol{\varphi}(\mathbf{x})-b \leq \varepsilon+\xi_{i}, \quad i=1,2, \cdots, N \\ \mathbf{w}^{T} \boldsymbol{\varphi}(\mathbf{x})+b-y_{i} \leq \varepsilon+\xi_{i}^{*}, \quad i=1,2, \cdots, N \\ \xi_{i}, \xi_{i}^{*} \geq 0, \quad i=1,2, \cdots, N\end{array}\right.$

where $\xi=\left[\begin{array}{lllll}\xi_{1} & \xi_{2} & \ldots & \xi_{N}\end{array}\right]^{T}$ and $\xi^{*}=\left[\begin{array}{llll}\xi_{1}^{*} & \xi_{2}^{*} & \ldots & \xi_{N}^{*}\end{array}\right]^{T}$ are slack variables representing the upper and lower constraints on the outputs of the system, respectively. Both slack variables have positive values.

The constrained optimization problem can be solved by applying the Lagrange multiplier technique and the quadratic programming technique, and the regression 


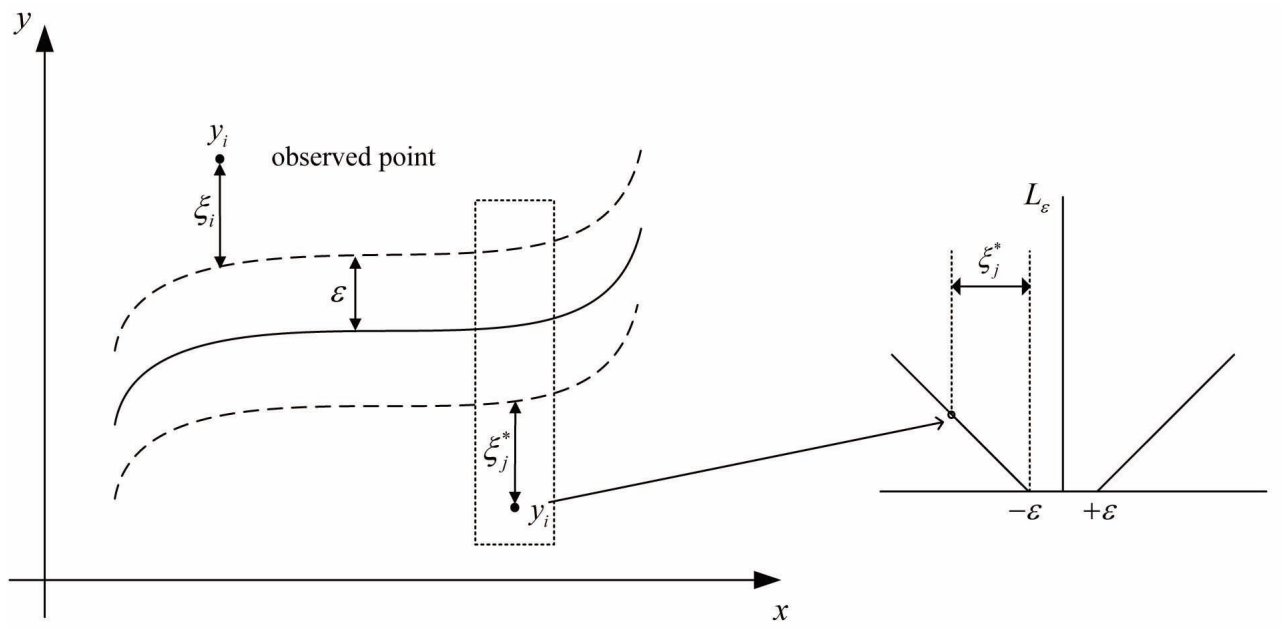

Fig. 5. Soft Margin Loss Setting for SVR.

function of Eq. (1) is expressed as follows:

$$
y=f(\mathbf{x})=\sum_{i=1}^{N}\left(\alpha_{i}-\alpha_{i}^{*}\right) \boldsymbol{\varphi}^{T}\left(\mathbf{x}_{i}\right) \boldsymbol{\varphi}(\mathbf{x})+b=\sum_{i=1}^{N} \beta_{i} K\left(\mathbf{x}, \mathbf{x}_{i}\right)+b,
$$

where $K\left(\mathbf{x}, \mathbf{x}_{i}\right)=\varphi^{T}\left(\mathbf{x}_{i}\right) \varphi(\mathbf{x})$ is called the kernel function. A number of coefficients $\beta_{i}$ are nonzero only for a subset of the training data. The training data points, which correspond to nonzero $\beta_{i}$, are called support vectors and have an approximation error $\geq \varepsilon$. The kernel functions used in this study were the following radial basis functions:

$$
K\left(\mathbf{x}, \mathbf{x}_{i}\right)=\exp \left(-\frac{\left(\mathbf{x}-\mathbf{x}_{i}\right)^{T}\left(\mathbf{x}-\mathbf{x}_{i}\right)}{2 \sigma^{2}}\right) .
$$

The kernel function parameter determines the sharpness of the radial basis kernel function. The bias $b$ is calculated as in [10]:

$$
b=-\frac{1}{2} \sum_{i=1}^{N}\left(\alpha_{i}-\alpha_{i}^{*}\right)\left(K\left(\mathbf{x}_{r}, \mathbf{x}_{i}\right)+K\left(\mathbf{x}_{s}, \mathbf{x}_{i}\right)\right),
$$

where $\mathbf{x}_{r}$ and $\mathbf{x}_{s}$ are the support vectors.

The two most relevant design parameters for the SVR model are the insensitivity zone $\varepsilon$ and the regularization parameter $\lambda$. An increase in parameter $\lambda$ penalizes the larger errors, which leads to a decrease in approximation error. This can be achieved only by increasing the vector norm of the weights. On the other hand, an increase in the vector norm does not ensure good generalization performance of a model, a condition known as the overfitting problem. Parameter $\varepsilon$ determines the size of the insensitivity zone. An increase in the insensitivity zone $\varepsilon$ means a decrease in the requirements for the accuracy of the approximation and it decreases the number of support vectors, leading to data compression. In addition, an increase in $\varepsilon$ affects the modeling of noisy polluted data, i.e. an increase in $\varepsilon$ causes a noisy polluted portion of data to be ignored.

\subsection{Selection of Training Data}

Each dataset was categorized as a training dataset, a verification dataset, or a test dataset. Appropriate selection of the training data is quite important because it can affect the performance of the SVR model. An SVR model can be well trained when data that includes a great deal of information is used. In this paper, a subtractive clustering (SC) scheme was used to select the training dataset [11]. The SC scheme introduces the concept of information potential. The information potential of each data point indicates the information quantity, and it is expected that a data point with high information potential has a large amount of information. The information potential of each data point is defined as a function of the Euclidean distances to all other input data points [11].

$$
P_{1}(k)=\sum_{j=1}^{N} e^{-4\left\|\mathbf{x}_{k}-\mathbf{x}_{j}\right\|^{2} / r_{\alpha}^{2}}, k=1,2, \ldots, N,
$$

where a positive constant $r_{\alpha}$ is a radius cluster defining a particular neighborhood of the cluster. The potential of a data point to be a cluster center is higher when it is surrounded by a substantial amount of neighboring data. After calculating the potential of each data point, the data point with the highest potential will be the first cluster center, and a cluster center can be obtained each time. In general, after determining the $i^{\text {th }}$ cluster center $\mathbf{c}_{i}$ and its potential value $P_{i}^{c}$, the potential of each data point is recalculated for all other data points excluding the effect of the first cluster center according to the following equation:

$$
P_{i+1}(k)=P_{i}(k)-P_{i}^{c} e^{-4\left\|\mathbf{x}_{k}-\mathbf{c}_{i}\right\|^{2} / r_{\beta}^{2}}, k=1,2, \ldots, N,
$$

where a positive constant $r_{\beta}$ denotes the radius cluster of the neighborhood. In addition, $r_{\beta}$ is normally greater than $r_{\alpha}$ to limit the number of clusters generated. When the potentials of all the data points were recalculated using Eq. 
(6), the data point with the highest potential was selected as the $(i+1)^{\text {th }}$ cluster center. The calculation stops if $P_{i}^{c}<\varepsilon P_{i}^{c}$ or specified data is selected; otherwise, the calculation continues. If the calculation stops at an iterative $\operatorname{step} N_{c}$, there are $N_{c}$ cluster centers. The input/output data positioned in the cluster centers were selected to train the SVR model.

\section{UNCERTAINTY ANALYSIS}

The development of data-based models requires an uncertainty analysis to explain the accuracy of their predictions. Through uncertainty analysis, a PI can be calculated such that the precise value exists inside the PI at a specified confidence level.

Data-based models have several possible sources of uncertainty in the predicted values: the selection of training data, model structure including complexity, and noise in the input and output variables [12]. Since a data-based model was developed using a given training dataset, each possible training dataset selected from the entire population of data will generate a different model and there will be a distribution of predictions for a given set of observation data. In addition, model misspecification occurs when the model structure is incorrect, thereby introducing bias. To predict the PIs, this paper uses an analytical uncertainty analysis method [12-16].

The following regression models of Eq. (6) can be established from the $N$ training data points $\left\{\left(\mathbf{x}_{1}, y_{1}\right),\left(\mathbf{x}_{2}\right.\right.$, $\left.\left.y_{2}\right), \cdots,\left(\mathbf{x}_{N}, y_{N}\right)\right\}$ :

$$
y_{k}=f\left(\mathbf{x}_{k}, \boldsymbol{\theta}\right)+\varepsilon_{k}
$$

where

$$
\boldsymbol{\theta}=\left[\begin{array}{llll}
\theta_{1} & \theta_{2} & \cdots & \theta_{p}
\end{array}\right] .
$$

Here, $p$ is the number of parameters to be optimized. For a regression model of observation data $\mathbf{x}_{o}$, which is not part of the training data, the output prediction can be given by

$$
\hat{y}_{0}=f\left(\mathbf{x}_{0}, \hat{\boldsymbol{\theta}}\right) \text {. }
$$

Using the Taylor series expansion of the output prediction, the output prediction can be approximated to the first order as follows:

$$
\hat{y}_{0} \approx f\left(\mathbf{x}_{0}, \boldsymbol{\theta}\right)+\mathbf{f}_{0}^{T} \cdot[\hat{\boldsymbol{\theta}}-\boldsymbol{\theta}]
$$

where

$$
\mathbf{f}_{0}^{T}=\left(\begin{array}{llll}
\frac{\partial f\left(\mathbf{x}_{0}, \boldsymbol{\theta}\right)}{\partial \theta_{1}} & \frac{\partial f\left(\mathbf{x}_{0}, \boldsymbol{\theta}\right)}{\partial \theta_{2}} & \cdots & \frac{\partial f\left(\mathbf{x}_{0}, \boldsymbol{\theta}\right)}{\partial \theta_{p}}
\end{array}\right) .
$$

Using Eq. (11) replaced with $\mathbf{x}_{o}$ and Eq. (13), the prediction error can be calculated as follows:

$$
y_{0}-\hat{y}_{0}=\varepsilon_{0}-\mathbf{f}_{0}^{T} \cdot[\hat{\boldsymbol{\theta}}-\boldsymbol{\theta}],
$$

The variance of the prediction error can be expressed as

$$
\operatorname{Var}\left(y_{0}-\hat{y}_{0}\right)=\operatorname{Var}\left(\varepsilon_{0}\right)+\operatorname{Var}\left(\mathbf{f}_{0}^{T} \cdot[\hat{\boldsymbol{\theta}}-\boldsymbol{\theta}]\right),
$$

where

$$
\varepsilon_{0} \sim N\left(0, \sigma^{2}\right) \text { and }[\hat{\boldsymbol{\theta}}-\boldsymbol{\theta}]=N(0, \mathbf{S}) .
$$

In the SVR model, since the parameter $\boldsymbol{\theta}$ is not solved explicitly and is calculated implicitly with a standard quadratic programming technique to minimize the constrained risk function of Eq. (4), the variance-covariance matrix $\mathbf{S}$ cannot be calculated. On the other hand, the optimized parameters are not expected to be significantly different from the parameters determined from the minimization of squared errors. Therefore, if the parameter is assumed to be estimated properly with the well-known squared error minimization technique, the variancecovariance matrix can be estimated as follows [16]:

$$
\mathbf{S}=s^{2}\left(\mathbf{F}^{T} \mathbf{F}\right)^{-1},
$$

where

$$
\begin{aligned}
& s^{2}=\frac{1}{N-p} \sum_{k=1}^{N}\left(y_{k}-f\left(\mathbf{x}_{k}, \hat{\boldsymbol{\theta}}\right)\right)^{2}, \\
& \mathbf{F}=\frac{\partial \mathbf{y}}{\partial \boldsymbol{\theta}}=\left[\begin{array}{llll}
\frac{\partial \mathbf{y}}{\partial \theta_{1}} & \frac{\partial \mathbf{y}}{\partial \theta_{2}} & \cdots & \frac{\partial \mathbf{y}}{\partial \theta_{p}}
\end{array}\right], \\
& \frac{\partial \mathbf{y}}{\partial \theta_{i}}=\left[\begin{array}{llll}
\frac{\partial y_{1}}{\partial \theta_{i}} & \frac{\partial y_{2}}{\partial \theta_{i}} & \cdots & \frac{\partial y_{N}}{\partial \theta_{i}}
\end{array}\right]^{T} .
\end{aligned}
$$

The parameter vector $\boldsymbol{\theta}$ in SVR models consists of the parameters $\beta_{i}$ and $b$ of Eq. (6). The matrix $\mathbf{F}$ is called the Jacobian matrix of the first order partial derivatives in regard to the parameters determined from the minimization of squared errors.

The variance of the predicted output can be estimated as follows [16]:

$$
\operatorname{Var}\left(y_{0}-\hat{y}_{0}\right) \approx \sigma^{2}+\mathbf{f}_{0}^{T} \mathbf{S f}_{0} \approx s^{2}+s^{2} \mathbf{f}_{0}^{T}\left(\mathbf{F}^{T} \mathbf{F}\right)^{-1} \mathbf{f}_{0} .
$$

In addition, an estimate with a $95 \%$ confidence interval can be written as

$$
\hat{y}_{0} \pm 2 s \sqrt{1+\mathbf{f}_{0}^{T}\left(\mathbf{F}^{T} \mathbf{F}\right)^{-1} \mathbf{f}_{0}}=\hat{y}_{0} \pm \delta
$$

\section{APPLICATION TO COLLAPSE MOMENT ESTIMATION}

Overall, the necessary data needs to be obtained to devise SVR models for estimating the collapse moment of wall-thinned pipe bends and elbows. Since there is no sufficient related field data of nuclear power plants, the FEA data was used to develop SVR models for each loading condition and defect geometry case.

The data provided consist of a total of 3712 inputoutput data pairs $\left(x_{1}, x_{2}, \cdots, x_{8}, y\right)$, which are composed of 1700 extrados defect location cases, 1700 intrados cases, and 312 crown cases. The characteristic of the collapse 
Table 2. Estimation Accuracy of the Collapse Moments by the SVR Models.

\begin{tabular}{|c|c|c|c|c|c|c|c|c|}
\hline \multirow[b]{2}{*}{$\begin{array}{c}\text { Defect } \\
\text { location }\end{array}$} & \multirow[b]{2}{*}{ Fitness } & \multirow{2}{*}{$\begin{array}{l}\text { Number } \\
\text { (No.) of } \\
\text { support } \\
\text { vectors }\end{array}$} & \multicolumn{3}{|c|}{ Training data } & \multicolumn{3}{|c|}{ Test data } \\
\hline & & & No. & $\begin{array}{c}\text { Relative } \\
\text { RMS } \\
\text { Error (\%) }\end{array}$ & $\begin{array}{l}\text { Relative } \\
\text { maximum } \\
\text { error }(\%)\end{array}$ & No. & $\begin{array}{c}\text { Relative } \\
\text { RMS } \\
\text { Error }(\%)\end{array}$ & $\begin{array}{c}\text { Relative } \\
\text { maximum } \\
\text { error }(\%)\end{array}$ \\
\hline Extrados & 0.5427 & 619 & 1300 & 0.3079 & 3.4162 & 170 & 0.2871 & 0.8924 \\
\hline Intrados & 0.5102 & 634 & 1300 & 0.3380 & 2.6821 & 170 & 0.3518 & 1.3423 \\
\hline Crown & 0.7233 & 178 & 250 & 0.1609 & 1.1020 & 30 & 0.2216 & 0.8244 \\
\hline Total & - & - & 2850 & 0.3125 & 3.4162 & 370 & 0.3142 & 1.3423 \\
\hline
\end{tabular}

moment differs considerably according to the three wallthinned defect locations of the extrados, intrados, and crown, represented by $x_{1}$. Therefore, the data can be classified into the three classes of defect locations and three SVR models are designed for the three classes, respectively. The input signals, $x_{2}$ through $x_{8}$, indicate the bend radius, bend angle, and wall thickness at the thinning defect, thinning length, thinning angle, internal pressure, and bending modes of closing and opening, respectively. $y$ is the FEA output, which indicates the collapse moment. Table 1 lists the ranges of input signals for the SVR models.

It is important to confirm that the proposed algorithm for the test data that was not used in the training stage. Therefore, the collapse moment data is divided into the training and test datasets that are used for the training and test, respectively. The training dataset comprises 1300 input-output data pairs for the extrados case, 1300 for the intrados case, and 30 for the crown case among the acquired data, all of which were selected using the SC scheme. The test dataset comprises 370 data points among the 3712 acquired data points (refer to Table 2). The uncertainty of the developed SVR models was analyzed with the test dataset.

Table 2 summarizes the estimation results of the collapse moments by the SVR models. For the training dataset, the relative RMS errors for extrados, intrados, and crown defects were $0.3079 \%, 0.3380 \%$, and $0.1609 \%$, respectively. For the test dataset, the relative RMS errors for extrados, intrados, and crown defects were $0.2871 \%, 0.3518 \%$, and $0.2216 \%$, respectively. The maximum error of the test data was $1.3423 \%$. These maximum errors are not as important as the relative RMS errors but are represented to show that the maximum value could be high under a specific operating condition.

Fig. 6 shows the errors between the actual collapse moments and estimated ones and their PIs. The PIs are quite narrow, which means that the predicted values are highly accurate. Fig. 7 shows that the estimated collapse moments are covered well by the PIs with the coverage probability shown in Table 3. In addition, from Table 3, the 5 data points among a total of 370 test data points

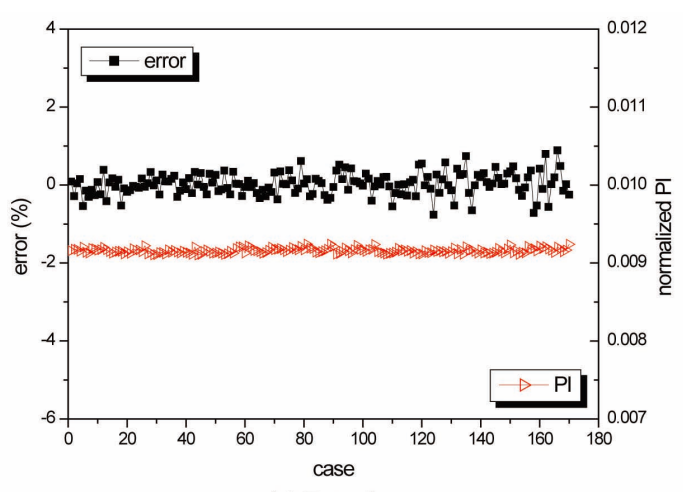

(a) Extrados

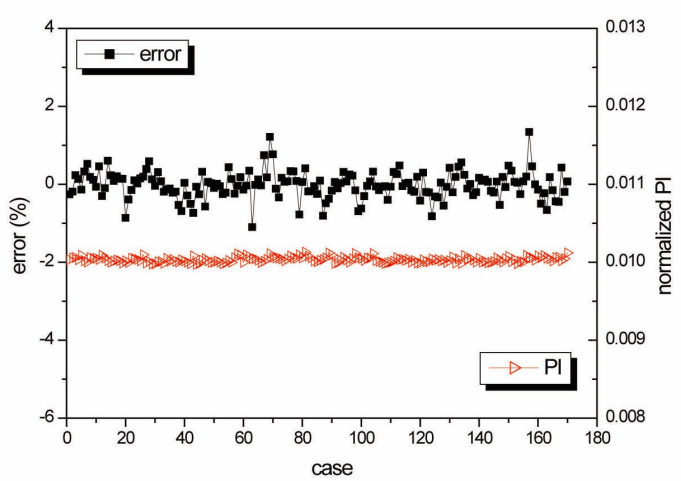

(b) Intrados

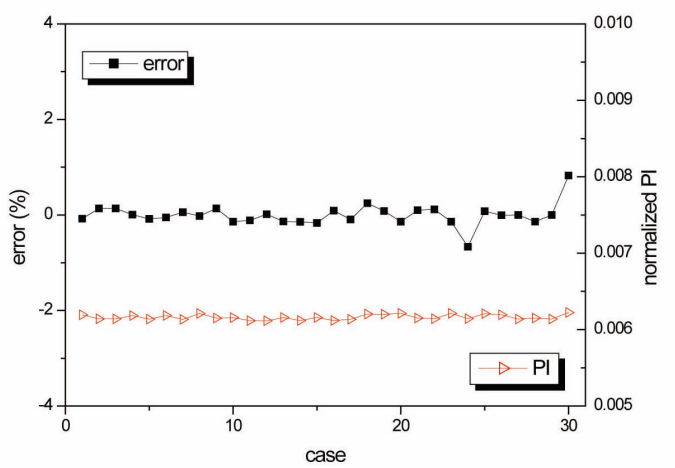

(c) Crown

Fig. 6. Errors between the Actual Collapse Moment and the Estimated One for the Test Data and PIs. 
Table 3. Coverage of the Pls.

\begin{tabular}{c|c|c|c|c}
\hline \multirow{2}{*}{$\begin{array}{c}\text { Defect } \\
\text { location }\end{array}$} & No. & $\begin{array}{c}\text { RMS } \\
\text { Error (\%) }\end{array}$ & Data number exceeding PI/test data number & Coverage Probability (\%) \\
\cline { 2 - 5 } & 170 & 0.2871 & $0 / 170$ & $100 \%$ \\
\hline Extrados & 170 & 0.3518 & $3 / 170$ & $98.24 \%$ \\
\hline Intrados & 30 & 0.2216 & $2 / 30$ & $93.33 \%$ \\
\hline Crown & 370 & 0.3142 & $5 / 370$ & $98.65 \%$ \\
\hline
\end{tabular}

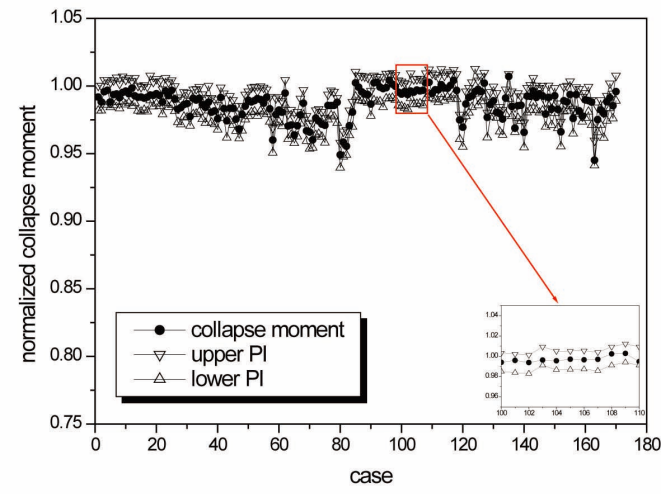

(a) Extrados

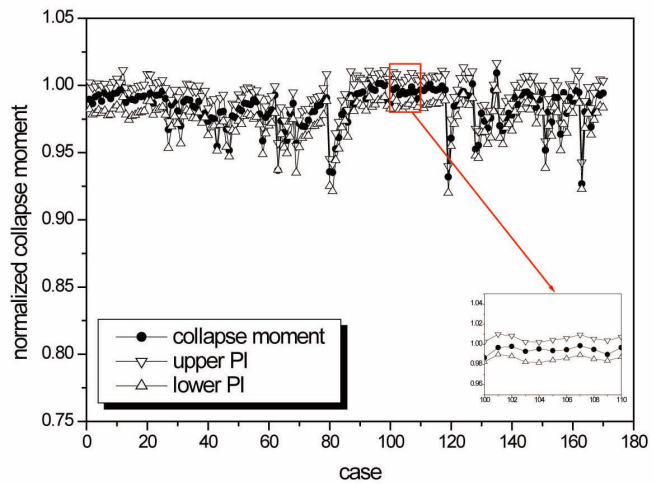

(b) Intrados

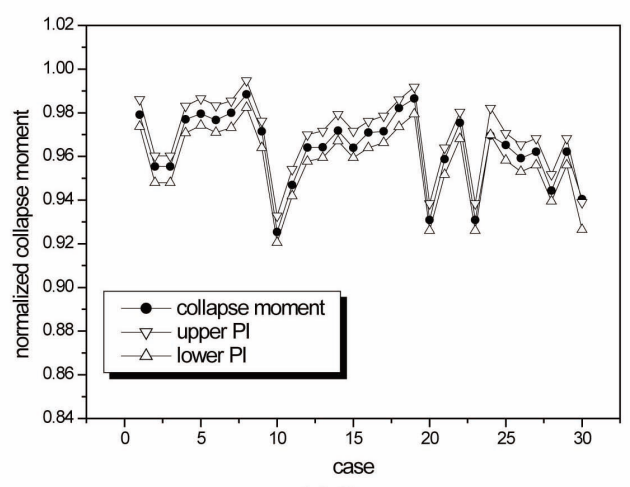

(c) Crown

Fig. 7. Coverage of PIs for the Test Data. miss the PIs of the analytical method. This indicates $98.65 \%$ coverage probability, which corresponds to the estimates that are solved with a $95 \%$ confidence level.

In this paper, FEA results and the used input data were assumed to be accurate. However, FEA results cannot be accurate, and the field input data may have measurement errors. Therefore, the uncertainty of the data-based model can increase when the field data are used.

\section{CONCLUSIONS}

Data-based modeling methods (SVR, FNN) were used in previous studies [4-5] to estimate the collapse moment due to the wall-thinned defects of bends and elbows in piping systems. Of the two methods, in this paper, uncertainty analysis was performed only for the SVR method because the SVR method has good estimation performance for the collapse moments compared to that of the FNN method. Three SVR models were developed for three datasets divided into the three cases of extrados, intrados, and crown defects. The data for training the SVR models was selected using a subtractive clustering scheme because informative data increases the learning effect. The uncertainty of the SVR models was analyzed using a test dataset independent of the training data. In the simulations of the test data, the RMS error was $0.3142 \%$ for the three cases. Estimates with a 95\% confidence interval were obtained for 370 test data points by performing analytic uncertainty analyses. The PIs were quite narrow, meaning that the predicted values were accurate. Therefore, the proposed SVR method can be used effectively to assess and validate the integrity of wall-thinned pipe bends and elbows.

\section{ACKNOWLEDGMENTS}

This work was supported through a grant funded by the Nuclear Research and Development Department of the Korea Institute of Energy Technology Evaluation and Planning (KETEP) by the Ministry of Knowledge and Economy of the South Korean government. 


\section{REFERENCES}

[1] V. C. Martzen and L. Yu, "Elbow Stress Indices Using Finite Element Analysis," Nucl. Eng. \& Des., vol. 181, pp. 257-265 (1998).

[2] M. A. Shalaby and M. Y. A. Younan, "Limit Loads for Pipe Elbows Subjected to In-Plane Opening Moments and Internal Pressure," J. Press. Ves. Tech., vol. 121, pp. 1723 (1999).

[ 3 ] B. Chexal, J. Horowitz, B. Dooley, P. Millett, C. Wood, and R. Jones, "Flow-Accelerated Corrosion in Power Plant," EPRI/TR-106611-R2 (1998).

[ 4 ] M. G. Na, J. W. Kim, and I. J. Hwang, "Collapse Moment Estimation by Support Vector Machines for Wall-Thinned Pipe Bends and Elbows," Nucl. Eng. Des., vol. 237, pp. 451-459 (2007).

[5] M. G. Na, J. W. Kim, and I. J. Hwang, "Estimation of Collapse Moment for the Wall-Thinned Pipe Bends Using Fuzzy Model Identification," Nucl. Eng. \& Des., vol. 236, pp. 1335-1343 (2006).

[6] K. Yahiaoui, D. G. Moffat, and D. N. Moreton, "Piping Elbows with Cracks, Part 2: A Parametric Study of the Influence of Crack Size on Limit Loads due to Pressure and Opening Bending," J. Strain Anal., vol. 35, pp. 35-46 (2000).

[7] K. Yahiaoui, D. G. Moffat, and D. N. Moreton, "Piping Elbows with Cracks, Part 2: Global Finite Element and Experimental Plastic Loads under Opening Bending," $J$. Strain Anal., vol. 35, pp. 47-57 (2000).

[8] H. D. Hibbitt, B. I. Karlsson, and E. P. Sorensen, "ABAQUS/Standard User's Manual," Hibbitt, Karlsson \&
Sorensen, Inc., Pawtucket, RI (2001).

[9] J. Chattopadhyay, "The Effect of Internal Pressure on InPlane Collapse Moment of Elbows," Nucl. Eng. Des., vol. 202, pp. 133-144 (2002).

[10] V. Vapnik, The Nature of Statistical Learning Theory, Springer, New York (1995).

[11] S. L. Chiu, "Fuzzy Model Identification Based on Cluster Estimation," J. Intell. Fuzzy Systems, vol. 2, pp. 267-278 (1994).

[12] J. W. Hines, B. Rasmussen, "Online Sensor Calibration Monitoring Uncertainty Estimation," Nuclear Technology, vol. 151, pp. 281-288 (2005).

[13] J. W. Hines and R. Seibert, "Technical Review of On-Line Monitoring Techniques for Performance Assessment," Volume 1: State-of-the-Art, NUREG/CR-6895, U.S. Nuclear Regulatory Commission, Washington, D.C (2006).

[14] J. W. Hines, D. Garvey, R. Seibert, and A. Usynin, "Technical Review of On-Line Monitoring Techniques for Performance Assessment," Volume 2: Theoretical Issues, NUREG/CR-6895, U.S. Nuclear Regulatory Commission, Washington, D.C (2007).

[15] J. W. Hines, J. Garvey, D. Garvey, and R. Seibert, "Technical Review of On-Line Monitoring Techniques for Performance Assessment," Volume 3: Limiting Case Studies, NUREG/ CR-6895, U.S. Nuclear Regulatory Commission, Washington, D.C (2008).

[16] R. Tibshirani, "A Comparison of Some Error Estimates for Neural Network Models," Neural Computation, vol. 8, pp. 152-163 (1996). 